\title{
LOS MODOS DE LA ATENCIÓN
}

The modes of attention

\author{
Daniel Eduardo Chaves Peña* \\ Instituto Caro y Cuervo, Bogotá, Colombia \\ aniel.chaves@caroycuervo.gov.co \\ Código Orcid: https://orcid.org/0000-0002-0912-5542 \\ JAIME YÁÑEZ CANAL \\ Universidad Nacional de Colombia, Bogotá, Colombia \\ jyanezc@unal.edu.co \\ Código Orcid: https://orcid.org/0000-000 1-9839-1 123
}

\begin{abstract}
Resumen
En este texto se analizan algunas concepciones clásicas y alternativas del fenómeno de la atención. Luego de revisar algunos de los planteamientos tradicionales a la hora de concebir este proceso cognitivo, se defiende la importancia de una perspectiva fenomenológica de la atención. En este sentido se retoman las ideas de fenomenólogos como Husserl, Sartre y Merlau-Ponty con el objeto de establecer una distinción de niveles en el proceso atencional. Específicamente, se distingue entre las formas pasivas y activas de la atención. La separación y caracterización de niveles es fundamental, pues permite establecer estratos del fenómeno que a menudo se pasan por alto en las teorías de la atención. La concepción fenomenológica de la atención postula distintos niveles de consciencia que ponen en evidencia las formas de significación de las que dispone el sujeto en su relación con el mundo: la forma pasiva se corresponde con la consciencia prerreflexiva, mientras que la activa puede vincularse a las formas reflexivas de dirigir la atención. En cuanto a las formas pasivas, se enfatiza en las formas de la organización de los contenidos de la experiencia, y en lo que concierne a las formas activas, se resalta cómo el sentido de agencia es fundamental en la determinación de la corriente de experiencias. Tales distinciones son fundamentales para una caracterización apropiada del campo de atención.
\end{abstract}

\section{Palabras clave \\ Atención, consciencia, fenomenología, prerreflexiva, activa, pasiva.}

Forma sugerida de citar: Chaves, Daniel \& Yáñez, Jaime (2021). Los modos de la atención. Sophia, colección de Filosofía de la Educación, 30, pp. 225-244.

* Investigador del Instituto Caro y Cuervo. Docente de la Maestría en Lingüística.

** Profesor Departamento de Psicología, Universidad Nacional de Colombia. Director del Grupo de Investigación "Estudios sobre Desarrollo Socio-Moral”. 


\begin{abstract}
In this text, it is claimed that a phenomenological approach to attention could provide important distinctions concerning different levels of consciousness. After criticizing some classical ideas about attention, the phenomenological ideas are introduced pointing how relevant they are for conceiving key aspects of attention that are usually overlooked in other theories. By revisiting seminal ideas from Husserl, Gurvistch, Sartre and Merlau-Ponty, the relationship between the workings of attention and the modes of consciousness explored by phenomenology is underscored. From this point of view, two basic modes of attention are distinguished: a passive form which is involved in the forms of synthesis responsible for the structure of the immediate contents of experience, and an active mode, characterized by the sense of agency which allows the subject to make distinctions, individuate and highlight different aspects from the structure of experience. There is a dynamic relation between these two forms of attention and how they can be identified with two modes of consciousness: while the passive form corresponds to pre-reflective consciousness, the active form is equivalent to the more reflective ways of directing the attention. The pre-reflective mode of consciousness characterizes the continuous forms of being related with the world without exerting meta-cognitive monitoring over our experiences. The active mode attention can only operate on the basis of pre-reflective consciousness.
\end{abstract}

Keywords

Attention, consciousness, phenomenology, pre-reflective, active, passive.

\title{
Introducción
}

La atención es entendida en posturas tradicionales como una carga de energía que se deposita en ciertos acontecimientos de acuerdo con la intensidad, extrañeza o novedad de estos eventos. El sujeto bajo esta mirada está sometido al vaivén de los acontecimientos del mundo y su percepción y consciencia son entendidos de manera reactiva. La conceptualización y el debate sobre este concepto de atención va apareada con nuevos abordajes sobre el problema de la consciencia. La consciencia es el problema que permite considerar al ser humano como un ser activo que establece unos parámetros para organizar y seleccionar la información que recibe y las maneras en como interactúa con su entorno.

Una teoría consistente de la consciencia debe incluir como un componente fundamental de su explicación un análisis exhaustivo de sus relaciones con la atención. En el fenómeno de la atención se manifiesta un aspecto esencial de la vida consciente: la dimensión activa de la mente en la capacidad de organización, selección y diferenciación de los contenidos de experiencia. Para poner de presente estas dimensiones de la experiencia consciente, es preciso criticar aquella concepción clásica de la atención que la reduce a una facultad psicológica que está encargada de albergar los fenómenos que vinculan al sujeto con el entorno, esto es, la captación de sensaciones en función de la intensidad o los contrastes cualitativos de los estímulos externos. 
La consciencia es el "problema duro", el problema más relevante en la investigación actual de las ciencias cognitivas, ya que pone en un primer plano el sentido de unidad de las experiencias y el sentido de agencia. El abordaje del fenómeno de la atención permitirá entender algunos de los debates sobre la conceptualización en torno a la consciencia y el aporte de las perspectivas fenomenológicas a la cuestión.

Este artículo se enfoca en uno de los aspectos fundamentales de la atención, esto es, el fenómeno de saliencia o prominencia de un contenido por encima de otros. Al analizar este fenómeno, el artículo intenta responder a los siguientes interrogantes ¿Qué es lo que ejerce la organización y selección de los contenidos de experiencia que son focalizados por la atención? ¿Se trata de un proceso reflexivo o implícito de consciencia? ¿Por qué la experiencia consciente no se agota en el objeto focalizado, sino que parece abarcar los otros contenidos potenciales de la atención? En el texto se abordan estas preguntas desde diferentes perspectivas y se toma posición con la propuesta fenomenológica, la que posibilita resaltar el papel activo de la subjetividad y las maneras en como los sujetos perciben de manera coherente su experiencia

\section{Crítica a la concepción clásica}

¿Por qué resulta insuficiente la caracterización del modelo clásico? El tipo de casos que se tiene en mente a la hora de pensar a la atención como la mera captación pasiva de las sensaciones son más bien casos bastante simples y extremos: por ejemplo, suele citarse la circunstancia en que un fenómeno inusual de repente irrumpe en la consciencia en virtud de su mera intensidad (e.g. el choque de un auto que produce un fuerte ruido y demanda que la atención se dirija a percibir tal evento). Atendiendo a este tipo de fenómenos, se pueden mencionar las investigaciones de la psicofísica de Hardy Leahey (1994) y Fechner (1966) y algunas versiones de la psicología cognitiva con los trabajos de Broadbent $(1954 ; 1958)$, de Conway et al. (2001) y Driver (2001), que estuvieron dirigidas a establecer las relaciones entre la intensidad de los estímulos y el momento o umbral de 'consciencia'. De acuerdo a lo afirmado, la conciencia en estas perspectivas se asociaba directamente al fenómeno de la atención, entendido como la energía psíquica que reacciona ante la intensidad de un estímulo. La conciencia opera en estos casos como un 'darse cuenta' de la ocurrencia de un acontecimiento. Sobre la base de estos fenómenos, la atención se entiende como una facultad psicológica que está abocada a la captación 
puramente receptiva de sensaciones que constituyen la materia prima sobre la que se realizan ciertas representaciones del mundo.

En oposición a estas visiones clásicas que veían la atención como la luz de una linterna que era dirigida por la saliencia de ciertos estímulos del mundo, se han propuesto en la psicología otras visiones que establecen un mecanismo interno de orientación de la atención. Obviamente no se puede desconocer que existe esta manera automática y bastante simple en el operar de la atención, la cual permite que los organismos puedan reaccionar a las variaciones del mundo circundante. En el mundo animal y en las formas más elementales del funcionamiento cognitivo existen ciertos estímulos que despiertan una determinada carga energética y que generan de manera rápida unas conductas reactivas, o unas respuestas automáticas. Este tipo de reacciones que se pueden denominar como expresiones de una atención pasiva, no son las únicas maneras en que la atención se expresa. A las formas de atención pasiva que actúan como fuerzas determinadas por el mundo circundante, o como eventos causales del mundo exterior, se suele oponer la atención activa que se origina desde el interior de la vida psíquica.

En el segundo grupo se incluye el fenómeno más común de la atención, a saber, los casos en los que deliberadamente se dirige la atención hacia los fenómenos que son interesantes para el agente cognitivo. Si, por ejemplo, en una clase de dibujo al aire libre se pide la representación de un árbol teniendo un modelo presente, la atención captará su forma, sus colores, el tipo de hojas, etc., de una manera tan detallada que no tendría lugar de no ser por el proyecto perceptivo que voluntariamente se ha trazado el dibujante. Es claro que sin la participación de un proyecto deliberado de atención activa, todas esas características y aspectos pasarían desapercibidos; pero en el momento en que se atiende activamente, la consciencia establece un horizonte de significación (en este caso, las propiedades cualitativas y morfológicas del percepto) donde es posible hacer descubrimientos cognoscitivos, o afinando la percepción, articular lo que se sabe acerca del árbol con lo que actualmente se contempla.

Sin embargo, esta manera de plantear la diferencia entre una atención reactiva o pasiva y un modo activo no puede ser el punto de partida para el análisis. En primer lugar, porque ni siquiera en el caso de las formas pasivas la atención opera reactivamente; la cuestión radica en que el choque del auto no tiene un valor per se, en virtud de su intensidad, para llegar a la consciencia. El hecho de que sea notado como algo que llama la atención depende de la situación en la cual se encuentre el sujeto: así, por ejemplo, al estar en una exhibición de Nascar o de Monster Trucks, el cho- 
que de los autos tal vez ya no sea una impresión que exija una reacción automática de la atención. Por lo tanto, no son las propiedades sensoriales del estímulo lo que garantiza su captación consciente, sino la situación o el contexto en que la consciencia establece un campo de experiencia en el cual es posible hacerse más sensible a los fenómenos circundantes.

Ahora bien, esta aclaración no implica que se abandone la distinción intuitiva entre un modo pasivo y uno activo de la atención, pues tal diferencia atañe al hecho de que algunos fenómenos simplemente irrumpen en la consciencia sin que exista una intención trazada de antemano que apunte a captarlo, mientras que otros, en cambio, se descubren o reconocen porque los precede una intención volitiva dirigida a ellos. Si se quiere entonces comprender esta diferencia fundamental en los modos de atención, sus características y funciones específicas, es necesario explorar en qué sentido esos modos de la atención se despliegan en un campo de consciencia.

\section{La atención y el campo de consciencia}

$\mathrm{Al}$ decir que la conciencia opera desde un campo u horizonte de significación en donde la atención se expresa, se busca resaltar la volatilidad y dinámica del operar psíquico y la presencia de dimensiones que pueden variar constantemente en la medida en que el sujeto se desenvuelve en su mundo. Esto se hace evidente cuando se parte del hecho de que la atención discurre continua y discontinuamente entre los múltiples contenidos de la experiencia: la atención puede estar dirigida a un evento del mundo externo; y al mismo tiempo considerar las actividades que deben desarrollarse durante el día, sin embargo, si algo inusual sucede en la calle y puedo entonces poner de relieve en mi consciencia lo que sucede en el campo perceptivo. Más que una función cognoscitiva, la atención refiere al modo de existencia de las experiencias en la corriente de consciencia. Así, las maneras en que se distribuye la atención en el discurrir de las experiencias determinan el modo de ser conscientes.

Este hecho ya había sido resaltado por W. James (1989; 2000), y constituye la base para la identificación de la atención con la dinámica y estructura del 'campo de consciencia'. La estructura del campo de la consciencia designa precisamente la organización mínima que hace que la atención fluya entre el contenido de experiencia focalizado y los contenidos que correlativamente se desplazan hacia el margen. La focalización de la atención en alguno de los contenidos y sus distintas propiedades 
tiene por efecto que los demás contenidos retrocedan momentáneamente hacia los márgenes del campo, sin que desaparezcan y sin que su presencia sea plenamente determinable.

Si se realiza un corte momentáneo sobre la corriente de consciencia, se puede notar que, de manera simultánea, hay múltiples contenidos que compiten por la atención: esto es lo que se tiene en mente cuando se dice, por ejemplo, 'no pude escuchar lo que decías porque estaba concentrado en la lectura'. Dependiendo de los intereses y las intenciones del momento, entre las distintas experiencias se genera una suerte de distribución jerárquica de la atención: esto es, que los modos del margen y el foco de atención se corresponden, respectivamente, con un menor o mayor grado de determinación en los contenidos de la experiencia. Al respecto resulta ilustrativo un ejemplo que proporciona Sartre (2006) para señalar el carácter global de la consciencia que no se agota en los contenidos focalizados: luego de haber estado leyendo durante todo el día, ya en la noche, cuando se empieza a experimentar algo de distracción, las imágenes actualmente representadas no se articulan con las pasadas, si bien no se siente cansancio; pero resulta que debido a la extensa jornada de lectura, se descubre finalmente que nuestros ojos se encuentran irritados. El dolor había permanecido en el margen del campo de consciencia, pero, no obstante, esta experiencia estaba configurando el contenido global de la consciencia en ese momento. El punto que quiere resaltar Sartre consiste en que la experiencia del dolor, a pesar de no ser focalizada, configura la consciencia global del momento.

Volviendo a la imagen que despierta un horizonte de percepción queda claro que los eventos compiten por atraer la atención del sujeto según lo que motive su experiencia en un momento dado, sin que la consciencia se entienda como el piloto que de manera predeterminada acompaña el desempeño variable de la atención. La consciencia tampoco ha de entenderse como un jinete que opera de manera reflexiva sobre los actos automáticos de percepción. Se hace énfasis en esta idea ya que para la perspectiva fenomenológica la consciencia no se identifica con un proceso de reflexión explícita que controle todos los procesos cognitivos. La consciencia, más bien, se entiende como una presencia constante que opera desde maneras prerreflexivas y que de manera particular determina la dirección de la atención y los procesos de reflexión y reporte explícito. Esta postura, de clara orientación fenomenológica, demanda un mayor espacio para su clarificación. 


\section{La consciencia prerreflexiva como forma básica de la consciencia}

Las posturas fenomenológicas no entienden la consciencia según procesos de reflexión, ni como formas de monitorio de un nivel superior o metacognitivo, ni como la expresión de actos de introspección. Contrario a esto la consciencia se entiende como parte de una experiencia primaria ${ }^{1}$.

Para Sartre (2006) la reflexión es una especie de consciencia derivada, es decir es el proceso cuando la consciencia se toma a sí misma como objeto. Esta consideración de la consciencia implica una diferenciación entre sujeto y objeto, lo que significa que el sujeto se distancie de sí mismo para analizarse desde una perspectiva de tercera persona. Para Sartre esta fragmentación de la misma consciencia conduciría a una regresión al infinito, ya que la consciencia que se analiza a sí misma necesita a su vez hacer una reflexión sobre ese mismo proceso de manera indefinida, o, para evitar lo anterior, debería suponer un proceso inconsciente ${ }^{2}$ inicial que posibilitaría el bucle de autorreflexión. Por estas razones la consciencia debe suponer varios niveles y una consciencia primaria, de naturaleza prerreflexiva.

Para que el sujeto pueda hacer una reflexión sobre su experiencia debe poseer una sensación de unidad, una sensación de que él es el sujeto de la experiencia. Esta es la consciencia prerreflexiva, una sensación de presencia del self consigo mismo en todas sus experiencias. Zahavi (2005) ilustra esta idea remitiéndose a una situación descrita por el mismo Sartre en la cual el sujeto completamente absorto en la lectura de una novela. Si la novela logra atrapar toda su atención, el sujeto puede incluso olvidarse de sí-mismo y de muchas otras cosas del mundo exterior. La lectura de la novela se lleva a cabo sin necesidad de reflexionar sobre este proceso, o la actividad que estamos realizando. Pero si intempestivamente aparece alguien e interrumpe al sujeto preguntándole al sujeto qué ha estado haciendo durante las últimas horas, este no tendrá ninguna dificultad en reportar que ha estado leyendo una novela. Con esta situación se puede entender que a pesar de que no se reflexione sobre el acto de leer, ni lo que la lectura produce, el sujeto siempre puede saber que está siempre presente en su experiencia. Nuestro self ha estado en la experiencia presente sin que tenga que verse a sí mismo como un objeto de reflexión desde una perspectiva de tercera persona.

De acuerdo con este ejemplo se puede entender la diferenciación que hace Sartre entre la consciencia prerreflexiva y la reflexiva ${ }^{3}$. La primera es ese autoconocimiento en el que el sujeto siempre está inmerso. 
La segunda, la consciencia reflexiva, es ese proceso de reflexión donde el sujeto puede referirse a sí mismo de la misma manera en que puede referirse a los objetos del mundo.

Por supuesto la consciencia prerreflexiva, al ser primaria, está presente siempre y puede manifestarse sin que haya un proceso de reflexión o de monitoreo de segundo orden. En cambio, esta última capacidad metacognitiva demanda siempre la presencia de un sujeto que se percibe como una unidad siempre presente. Para Sartre "hay un cogito prerreflexivo que es la condición del cogito cartesiano" (citado por Zahavi, 2005, p. 30).

El concepto de atención debe entenderse de manera estrecha con esta caracterización de los niveles de consciencia. Normalmente el sujeto puede atender a una infinidad de eventos sin que tenga que reflexionar sobre su valor, o significado, y mucho menos plantearse algo de manera explícita que le asegure la coherencia o el encadenamiento de sus experiencias. Por supuesto, si este fluir prerreflexivo se ve alterado por algún evento inesperado, o no anticipado, la actitud reflexiva toma el comando de nuestras acciones para focalizar y reorganizar nuestro comportamiento.

\section{Origen y factores organizativos de la experiencia perceptiva}

Hasta ahora se ha mostrado de qué manera las experiencias que ocupan la corriente de consciencia requieren de un cierto grado de atención para existir en cuanto tales. Asimismo, se puso de relieve que las experiencias tienen lugar en el campo de consciencia en el modo primordial de la prerreflexividad, ya que no se precisa de un acto ulterior de reflexión para ser conscientes de las experiencias; es más, la consciencia prerreflexiva condiciona y motiva los actos de reflexión. No obstante, estos planteamientos todavía no alcanzan a aportar la clave de uno de los aspectos funcionales de la atención; tan solo señalan en qué sentido las experiencias existen con un cierto grado de atención. La función básica de la atención a la que consiste en hacer prominente o separar del flujo de la consciencia uno de los contenidos que se transforma así en el 'tema' de nuestro interés. ¿Qué hace posible que uno de los contenidos de la corriente de consciencia se ponga de relieve entre los demás? El tratamiento fenomenológico de la prominencia de un contenido permitirá delimitar de mejor manera lo que antes se ha denominado como los modos pasivos y activos de la atención.

El fenómeno de la prominencia de un contenido en el flujo de la consciencia atañe al problema de la organización de la experiencia sensible. De acuerdo con Carpintero (1996) y Driver (2001), en las teorías 
psico-físicas del siglo XIX se buscaba dar cuenta de este fenómeno señalando las diferencias cuantitativas, de contraste y semejanza entre los datos de sensación. Uno de los supuestos básicos de esta teoría consistía en lo que los teóricos de la Gestalt denominarán más tarde como la 'hipótesis de la constancia', la cual propone que existe una correspondencia uno a uno entre los estímulos físicos y las sensaciones: en la medida en que los estímulos sobrepasen un cierto umbral de intensidad, podrán ser notados en el nivel consciente. Ya en el primer apartado se mostraron algunas de las dificultades de esta teoría; en este punto es posible resumir el problema de la siguiente manera: hay fenómenos de la experiencia perceptiva que no tienen un correspondiente directo en el nivel de la intensidad o saliencia de los estímulos, y que por tanto requieren de la aceptación de una instancia o forma organizativa. Esto es lo que sucede, por ejemplo, en el fenómeno phi, donde se puede percibir un movimiento aparente entre dos luces que permanecen objetivamente separadas. Asimismo, es lo que se pone de presente en las imágenes ambiguas (el pato-conejo o la imagen que puede mostrar los dos rostros o una copa), donde la configuración del fenómeno perceptivo depende de la escogencia del contorno que delimita una u otra figura.

Como reacción a los supuestos atomistas del empirismo inglés que explicaban la organización de la experiencia como la mera adición de átomos de sensación, los planteamientos de James (1989), la fenomenología de Husserl (1986, 2001), la escuela de Graz (Meinong, 1981), y la teoría de Gestalt (Koffka, 1967; 1979) buscaron establecer los principios organizativos de la experiencia en distintos factores de la subjetividad. De manera rápida se puede decir, que a pesar de las coincidencias en su crítica al asociacionismo y a las posturas de la psicofísica, presentan diferencias radicales entre sí. La postura de James (1989) y la de la escuela de Graz (Meinong, 1981) se pueden agrupar en una misma tendencia ya que sostienen que la organización y diferenciación de la experiencia es algo impuesto extrínsecamente a la corriente de consciencia, ya sea en virtud de la atención activa (James) o por un acto de orden superior del intelecto (escuela de Graz). Por su parte, la postura de la fenomenología husserliana junto con la teoría de la Gestalt conforman otro grupo en la medida en que consideran que la organización de la experiencia es un aspecto inmanente de la sensibilidad o de la corriente misma de experiencias. Las diferencias entre estas posturas pueden pasarse por alto y, en su lugar, ampliar algunas ideas sobre la atención. Puesto que el texto está orientado a examinar el valor y el alcance de la atención activa y el proceso de diferenciación y organización de los contenidos de experiencia, 
se resaltarán algunas ideas de los autores citados para ir esclareciendo los puntos centrales en la clarificación de algunos aspectos de la consciencia.

En este punto se recurre a la caracterización que Gurwitsch (1979; 2009) realiza sobre James y las funciones que este le atribuye a la atención. La relevancia de esta caracterización radica en señalar cierto impase explicativo que enfrenta la teoría de James al asumir como principio de la organización de la corriente de experiencias la función selectiva de la atención. Al respecto, dice Gurwitsch (1979):

De acuerdo con James, aun cuando el 'campo de la consciencia' se encuentre organizado, no debe considerarse tal cosa como una característica primaria, auténtica y originaria de la experiencia. Y esto se debe a que la organización le vendría dada, superpuesta a la consciencia. James se mantuvo fiel a esta tesis durante toda su carrera' (p. 37).

En contra del atomismo de la explicación empirista, James (1989) considera que los componentes más básicos de la experiencia no son los átomos de sensación sino las denominadas 'totalidades sensibles'. Las totalidades sensibles designan el fluir temporal de las experiencias previo a cualquier acto de conceptualización; fuertemente influido por Bergson $(2006)^{4}$, James caracteriza la corriente de consciencia como un todo indiferenciado de estados 'fusionados'. Así pues, el problema para James no radica en cómo unas partes inconexas se unen en la mente para configurar un objeto coherente (lo que sería el problema de la consciencia para el empirismo inglés), sino más bien de qué manera la consciencia es capaz de disociar o separar de la masa informe de las totalidades sensibles los aspectos relevantes al agente cognitivo. Se entiende así la función que James le asigna a la atención activa: la organización y diferenciación del campo de consciencia es efecto de la actividad selectiva de la atención que, en el continuum de las totalidades sensibles, introduce segmentaciones y diferenciaciones del contenido.

¿Cuál es la dificultad que afronta la teoría de la atención activa de James? El punto central de la crítica de Gurwitsch se formula de la siguiente manera: no es posible que la atención separe y diferencie los contenidos de la experiencia si no posee de antemano una imagen de lo que se busca separar; es decir, que la organización de los contenidos de consciencia debe preceder los procesos de atención y de diferenciación de la información. Tratando de salvar esta dificultad, James propone que la atención haya actuado de manera azarosa en los primeros encuentros con los objetos. Parece suponer así, incluso yendo en contra de su tesis sobre la naturaleza volitiva de la mente, que el funcionamiento azaroso 
de la atención, junto con la repetición de la experiencia, llevaría a cabo el fenómeno de la separación sobre la corriente de consciencia. Al aceptar esta explicación, no se entiende cómo un conocimiento previo que ha sido aislado de manera azarosa por la atención pueda ser puesto de relieve nuevamente por un mecanismo que opera al azar. Esto resulta altamente improbable. Una vez que se aceptado que la atención selectiva opera al azar, entonces debe operar de igual manera en todos los casos. Igualmente, si dos cosas se presentan inicialmente al azar y son agrupados de determinada manera es porque en el sujeto debe existir una forma de organización previa que les dé sentido, o al menos los agrupe bajo particulares esquemas.

Además, de acuerdo con lo que plantea Köhler (1959; 1967), la influencia de la experiencia pasada sobre la actual no es suficiente para explicar el fenómeno de la prominencia. Las estructuras de camuflaje que ha analizado Köhler son pertinentes en este caso. Ciertas figuras (ver la figura A) contienen objetivamente a otras (ver figura a) que resultan familiares a partir de experiencias pasadas. En el primer grupo de figuras presentadas a continuación se puede ver cómo un determinado elemento se oculta en otra imagen. La E de la figura $a$ está oculta en la primera figura $(A)$. En cambio, en el otro grupo de figuras (de la derecha), la presencia de la figura $a$ en $A$ se hace patente. El 4 (de $a$ ) se observa sin dificultad en la primera figura de este grupo $(A)$.

Figura 1

\section{Estructuras de camuflaje}
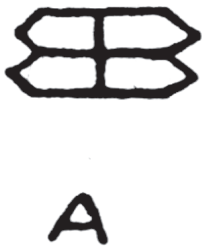
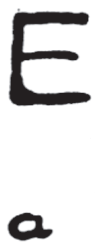

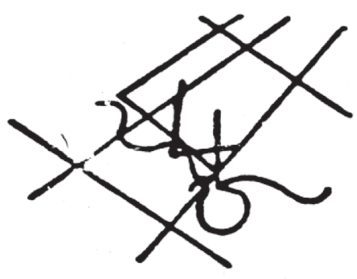

A

Fuente: Gurwitsch (1979, p. 43).

El hecho de que la figura $a$ se oculte o se haga manifiesta no depende entonces de que las figuras resulten familiares, pues tanto la $E$ como el 4 son igualmente familiares. Lo que se presenta en el campo perceptivo depende más bien de que las líneas que trazan la fisionomía gestáltica de 
la figura $a$ mantengan su estructura y no se fusionen con las de la figura A. De esta manera se entiende el punto de Gurwitsch (1979):

...es del todo imposible que la mera repetición y acumulación de experiencias tenga por resultado la separación misma de las unidades en cuestión. Para que estas puedan servir de vehículo al crecimiento por experiencia, es menester primero que se separen; es decir, que antes han de surgir del seno del campo y desprenderse unas de otras (p. 48).

Ahora bien, esto no significa que la experiencia previa no cumpla un papel esencial en el fenómeno de la prominencia de ciertos contenidos de consciencia. La réplica de Gurwitsch a James apunta a que la experiencia previa, al sedimentar conjuntos significativos, participa en lo que él denomina la 'fase exploratoria' de la constitución de la prominencia, esto es, que la búsqueda activa de una configuración suele invocar la función de la memoria en cuanto que el sujeto prueba distintas configuraciones que ya se han consolidado, tratando de hallar una resonancia con lo dado en el campo perceptivo. La 'fase exploratoria' se distingue de la 'fase de logro', la cual designa la puesta en relieve de una configuración por encima de otras, esto es, el momento en que la figura finalmente se destaca sobre el fondo. De acuerdo con Gurwitsch, este proceso de prominencia opera en el nivel más básico de la organización sensible, y es el fenómeno que antecede a la captación que lleva a cabo la atención selectiva. Dicho en los términos del apartado anterior, la organización y diferenciación del campo perceptivo tiene lugar en el plano más básico de la experiencia prerreflexiva.

Teniendo en cuenta estas dificultades de la teoría de James, Gurwitsch (2009) intenta dar cuenta de lo que sería el origen y los factores de organización inmanentes a la corriente de consciencia mediante un recurso a la teoría de la Gestalt y la fenomenología husserliana. En el estudio de configuraciones de puntos, líneas, curvas y figuras más complejas, los teóricos de la Gestalt propusieron cuatro factores que contribuyen al fenómeno de la organización de las partes en conjuntos unitarios: 1) La proximidad; 2) La igualdad; 3) El cerramiento y 4) La continuación apropiada. Como bien lo ha señalado Merleau-Ponty (1984), aunque estos factores se han interpretado dentro de la teoría de la Gestalt como las condiciones objetivas de la organización de los estímulos, admiten no obstante una lectura fenomenológica, esto es, en tanto que aspectos estructurales que son intrínsecos a las estructuras perceptivas experienciadas ${ }^{5}$. 


\section{La atención y las síntesis pasivas}

Es preciso resaltar la importancia de los fenómenos del 'cerramiento' y de la 'continuación apropiada'. Aunque estos factores fueron establecidos especialmente en el terreno de la experiencia visual, su importancia desborda este ámbito. La importancia del factor de la 'continuación apropiada' se hace evidente en los casos de estructuras temporales de la experiencia, esto es, estructuras que exigen un desenvolvimiento conclusivo (e.g. una conversación o una melodía). En la fenomenología husserliana (Husserl, 1986; Zahavi, 2005), la temporalidad constituye la síntesis pasiva ${ }^{6}$ fundamental de toda experiencia. Esto es evidente en el caso de la melodía, cuya experiencia unificada no sería posible si la consciencia estuviera abocada a la captación de las notas presentes. En efecto, para tener la experiencia del desenvolvimiento de una melodía, es preciso que la consciencia no solo retenga las notas previas, sino que también, a partir de las notas escuchadas, anticipe y prefigure un posible desenlace que caracteriza la contextura misma de la melodía. La retención, entendida como consciencia de lo recién pasado, el momento de la impresión presente y cierta medida de anticipación constituyen el horizonte temporal que garantiza la experiencia de un fenómeno unitario de sentido.

Se trata de síntesis pasivas porque la unificación se da en el nivel de la experiencia prerreflexiva, sin que tenga que participar un acto de orden superior que opere la unificación de las notas: la síntesis se despliega en el plano de la pura sensibilidad. Es preciso anotar que la temporalidad, si bien es primordial, no es la única síntesis pasiva. Husserl trabaja de manera semejante las síntesis de homogeneidad, heterogeneidad, contraste, semejanza y orden, las cuales constituyen el terreno de las investigaciones fenomenológicas del contenido sensible; son aquello que posibilita en el terreno de la afectividad prerreflexiva las tematizaciones de la consciencia explícita, también denominadas como síntesis activas de la consciencia.

De este modo, al no aceptar que haya procesos de prominencia y diferenciación que surgen de la experiencia misma, la teoría de James se cierra a una explicación del origen de la organización y se ve abocada a imponer extrínsecamente lo que es un aspecto inmanente de la corriente de experiencia: su carácter proto-diferenciado en función de síntesis pasivas de la sensibilidad que preparan el terreno para las actividades 'selectivas de la atención'.

Así pues, la conciencia se expresa a través de diferentes estados o niveles dentro de un horizonte que fluye y que se expresa en toda experiencia. La forma o nivel más básico de la conciencia es el modo prerreflexivo que todo lo determina y que siempre acompaña toda experiencia. 
Esta conciencia prerreflexiva es una primera forma en cómo se entienden las nociones de atención pasiva y activa, pero esta a su vez demanda unas mayores especificidades. Volvamos a ciertos fenómenos ya expuestos.

Es un acontecimiento casi común que en medio de un flujo de experiencias más o menos homogéneo (e.g. una conversación), algo invoque la atención (una melodía) sin que se haya estado pendiente de lo que ocurría alrededor. O el fenómeno de coctel, del que hablan los estudiosos de la percepción (Driver, 2001), que consiste en cambiar el foco de atención de una conversación con un interlocutor hacia otra conversación que desarrolla un tema de nuestro para el sujeto, o que nombra tan solo un personaje afín a sus simpatías. ¿Se habría oído algo sin haber tenido la intención de escuchar? $\mathrm{O}$, con otras palabras, ¿cómo se oye algo que no se estaba atendiendo? ¿Cómo algo llama la atención, sin haber sido parte de nuestro foco atencional? De acuerdo con lo que hemos establecido anteriormente, resulta verdadero decir que los objetos invocan nuestra atención sin que exista previamente un acto deliberado de la atención. La dirección de la atención no es azarosa en estos casos, sino que obedece a formas organizativas y selectivas que operan en el nivel de la experiencia prerreflexiva. Aquello que repentinamente invoca la atención está sujeto a una corriente de experiencias que obran como un horizonte unificado y coherente (la melodía, o el tema tratado por un par de contertulios vecinos, se convierte en el foco de la atención por cuanto que la parte que actualmente se despliega está vinculada a los intereses afectivos y a la historia de experiencias del sujeto en cuestión). En este punto la consciencia se vuelca hacia la parte melodiosa que actualmente inunda el campo de consciencia, pero, como ya lo habíamos señalado, esta percatación no se reduce al nivel del momento presente; la parte melodiosa reactiva las partes que antes no se habían atendido y que no obstante estaban presentes en el nivel de la experiencia prerreflexiva. Aparece así ante la consciencia el fenómeno como un fenómeno unitario de sentido.

El caso expuesto es un ejemplo de la percatación repentina de un contenido de experiencia al que no le precede un proyecto voluntario de búsqueda. En este caso es posible afirmar que, en la medida en que la atención está dirigida a otra parte, la conversación no-escuchada solo alcanza el estatus de un ruido blanco para la consciencia. No obstante, en el momento en que una secuencia de sonidos, en virtud de mecanismos y operaciones que escapan a la consciencia explícita, hace que la atención se vuelque sobre lo que se oía como ruido blanco y ahora, por el acto pasivamente motivado, se torna en una secuencia unificada cuyo significado es directamente asequible. Es en este sentido que como operan, en el nivel de la experiencia 
prerreflexiva, formas de unidad y síntesis pasivas que preparan el terreno de las elecciones o tematizaciones explícitas de la consciencia.

\section{El lugar de la atención activa en la experiencia}

En el apartado anterior se puso de relieve la importancia de las síntesis pasivas en la organización y diferenciación de los contenidos sensibles. Pero este es solo un componente de la investigación, a saber, lo que posibilita un modo involuntario de la atención. Es el momento de mostrar el papel de la dimensión activa de la consciencia en la síntesis de los fenómenos perceptivos, esto es, cuando de manera deliberada se traza un proyecto perceptivo. En este punto cabe resaltar el grado de control y auto-determinación que introduce la atención activa en el flujo de las experiencias. Ante el bombardeo continuo de datos sensoriales sobre los sentidos, la atención activa tiene el poder de invocar, sobre la base de una experiencia sedimentada, un objeto interno, el cual crea una especie de vacío atrayente, sobre el que viene a diferenciarse el objeto o el aspecto deseado. Pero este proceso de atención selectiva no solo consiste en seleccionar ciertos eventos que se ofrecen a la percepción, sino en organizar y dar cuenta de ciertos detalles según sea el conocimiento y experiencia de ellos. Esto se hace evidente si se enfrenta a dos personas, con diferente habilidad y formación musical, a una determinada melodía. Resulta obvio que solo el experto descubrirá en la melodía los componentes armónicos, de ritmo, las figuras musicales que esta describe y el rango de octavas en el cual se desarrolla, mientras que el sujeto inexperto apenas distinguirá la secuencia de notas que caracteriza a la melodía. En este caso la atención opera como una especie de rejilla diferenciadora y organizadora de las sensaciones.

Los dos últimos ejemplos permiten formular dos formas en que se presenta la atención y las maneras en que el conocimiento adquirido y la experiencia previa pueden orientar la acción de maneras que relacionaríamos con la dimensión activa de la mente. Estos dos ejemplos se pueden diferenciar según sea el grado en que el sujeto dirija sus procesos de percepción y las maneras explícitas en que orienta su acción. Siguiendo en este punto a O'Shaughnessy (2002), las propiedades que introduce la dimensión activa de la consciencia en la organización de la experiencia perceptiva son las siguientes: (i) estructuración espacio-temporal de los fenómenos, (ii) individuación de aspectos u objetos del contenido perceptivo, (iii) inteligibilidad de los contenidos de experiencia individuados. 
Se presentan a continuación las propiedades de la atención activa en el caso de la experiencia visual: (i) cuando se dice que la atención activa imprime una estructuración espacio-temporal a los fenómenos que toma por objeto, se quiere dar a entender que al contenido en cuestión se le proporciona un marco de anclaje espacio-temporal en el cual se despliega como un fenómeno unitario de sentido. En contraposición a la actitud pasiva de la experiencia visual, la atención activa introduce una diferenciación y una organización espacio-temporal que el fenómeno no habría tenido de no ser por la intención voluntaria de mirar el espectáculo visual. En la actitud activa de la experiencia visual, los contenidos del campo visual adquieren un grado de diferenciación y determinación espacial característicos: por ejemplo, cuando se realiza un recorrido en un auto por un lugar de la ciudad poco conocido, se tiende a configurar una 240 representación de las relaciones de distancia o de tamaño de los edificios que servirán al propósito de una orientación futura en ese espacio.

La propiedad (ii) se deriva en cierto sentido de la primera. La individuación' de propiedades y objetos perceptivos se hace posible por cuanto que una intención perceptiva global (querer escuchar una melodía en cuanto melodía) introduce un marco de síntesis de las fases temporales del objeto en cuestión. Es preciso que la atención, en la medida en que sigue el despliegue de la melodía, retenga las fases pasadas y anticipe de alguna manera las notas siguientes, y solo de esa manera podemos decir que escuchamos una melodía.

La propiedad (iii) de la 'inteligibilidad' apunta a que la puesta en marcha de una actividad perceptiva intencional da lugar a que la razón y el entendimiento actúen junto con el conocimiento adquirido, para garantizar el reconocimiento a lo largo del tiempo de un objeto perceptivo uniforme.

Es en la ejecución de un proyecto intencional perceptivo que se ponen de presente las propiedades de la atención activa en la configuración de los contenidos perceptivos. La intención perceptiva global pone en juego una capacidad de síntesis de lo dado en la percepción; sin esa intención no habría contenidos individuados en el espacio y en el tiempo, pues todo fenómeno perceptivo requiere de una síntesis de sus fases pasadas, actuales y futuras. Precisamente en el caso de la percepción, la atención tiene la función de anclar al sujeto en proyectos perceptivos que delimitan horizontes espacio-temporales en los cuales puede realizarse una ampliación del mapa cognitivo de la realidad, pues la atención posibilita la individuación de un contenido en la medida en que se articula lo dado en la percepción con el conocimiento previamente adquirido. 
Si se está comprometido en un proyecto perceptivo activo (e.g. mirar y detallar el monumento), es claro que la intención actúa a la manera de un agente que sintetiza los distintos perfiles del monumento como pertenecientes a un solo objeto. Así es posible llegar a saber cuál es su forma y sus distintas apariencias dependiendo de la orientación y los desplazamientos a su alrededor. Asimismo, el proyecto intencional del atender activo introduce una especificación de las propiedades espaciales del objeto y las relaciones que mantiene con el lugar y los demás objetos que lo circundan; son estas características de estructuración y diferenciación aportadas por la atención activa las que garantizan la inteligibilidad del fenómeno no solo como un evento perteneciente a una corriente de consciencia, sino también como un objeto que pertenece a cierto tipo general de objetos (monumentos) que presentan tal o cual forma, es decir, como un objeto que se puede reconocer e individuar en distintas situaciones.

Solo un proyecto de atención activa proporciona una base firme para la constitución de un conocimiento duradero en la memoria, de tal suerte que si se pide un reporte pormenorizado del monumento visitado, el agente cognitivo estaría en la capacidad de reportar con una suficiencia de detalles las características del fenómeno en cuestión. Es esta la medida de orden y sentido que la atención activa introduce en la experiencia perceptiva.

\section{Conclusiones}

Este excurso sobre algunas de las modalidades y propiedades de la atención se ve justificado por la falta de una aclaración de las dimensiones activas y pasivas de la misma. El uso de los términos activo y pasivo para caracterizar los modos de la atención resulta inadecuado si, en primer lugar, la pasividad se concibe como la mera reacción a las variaciones de intensidad de los estímulos, pues de acuerdo con lo que se ha planteado, el fenómeno más básico de la atención, a saber, la prominencia de un contenido por encima de otros, obedece o se hace posible en función de un conjunto de estructuras organizativas de la consciencia. De este modo la aclaración fenomenológica de la dimensión pasiva enseña que la atención opera en virtud de la organización y diferenciación de los contenidos de la experiencia prerreflexiva.

$\mathrm{Al}$ aclarar los límites de lo que se ha denominado 'atención pasiva', queda el camino abierto para caracterizar su modo activo. Las estructuras de consciencia de la atención pasiva proporcionan el subsuelo para una potencial individuación del contenido, pero es solo en el plano de la acti- 
vidad de la consciencia donde se ejerce actualmente la posibilidad de diferenciar un contenido en la medida en que se articula con las intenciones del sujeto y el sistema de conocimiento ya adquirido. Asimismo, es en el plano activo donde se pone en escena la capacidad de agencia y de control por parte del sujeto en la elección de un contenido. Una manera de avanzar en la investigación del sentido de agencia que caracteriza buena parte de la experiencia subjetiva tendría entonces que profundizar sobre las maneras en que opera la atención activa, el modo en que la atención se articula con el sistema de conocimiento del sujeto en función de una intención trazada de antemano. De igual manera el estudio de la agencia debe contemplar los diferentes momentos del desarrollo del niño para poder delimitar las múltiples relaciones que se establecen entre las diferentes formas de conocimiento y los cambios cualitativos en los procesos intencionales.

\section{Notas}

1 En cambio, para las teorías de orden superior, o posturas meta-cognitivas dentro del procesamiento de la información y la psicología del desarrollo tradicional (Carruthers, 2005; Lycan, 1995; Piaget, 1976; Vygotsky, 2000), lo que hace a un estado mental un estado consciente consiste en el acto de ser tomado como objeto por un segundo acto (introspección o reflexión); así por ejemplo, no seríamos conscientes de lo que percibimos hasta que un proceso meta-cognitivo tome por objeto al estado primario (en este caso, la percepción). En este contexto el sentido de reflexión es tomado en su sentido literal, como el reflejo de una imagen en el espejo. Un contenido es "reflejado" en un plano superior que a su vez es atendido. De este modo, lo que hace a un estado consciente es el acto de vinculación entre un estado meta-cognitivo $\mathrm{y}$ un estado primario.

2 Los fenomenólogos (Husserl, 1986; Sartre, 2006) se oponen a la idea de que la consciencia emerja a partir de un proceso reflexivo o monitoreo de nivel superior, pues esto conduce, inevitablemente, a un regreso al infinito: si los estados mentales son conscientes en el sentido de ser tomados como objetos intencionales por un segundo estado mental, entonces estos estados mentales de orden superior, si es que han de ser conscientes, también han de ser tomados como objetos por un tercer estado, y así hasta el infinito. Los defensores de la teoría de orden superior han respondido con el planteamiento de estados mentales no-conscientes (Carruthers, 1994, Lycan, 1995). Según estos autores, al aceptar la existencia de estados mentales no-conscientes la regresión se detiene. De esta manera, la percepción o el pensamiento de segundo orden sería no-consciente. No obstante, los fenomenólogos consideran que esta solución, al apelar a estados mentales no-conscientes, nos deja con un vacío explicativo, pues efectivamente, ¿Cómo es posible que dos estados mentales no-conscientes puedan hacer que uno de ellos se vuelva consciente? Sin duda es difícil concebir que de esa relación meta-cognitiva surjan las cualidades fenoménicas de la experiencia.

3 En la fenomenología la obra de Husserl tuvo varios cambios y varias reacciones de acuerdo con su período de desarrollo conceptual. Las primeras obras de Husserl, generaron reacciones en Heidegger, Sartre, Merleau-Ponty, Scheler y otros autores. 
Estos alegaban la necesidad de postular una instancia prerreflexiva como el aspecto central de la consciencia. Como se entendiera esta instancia prerreflexiva sería lo que habría de diferenciar a las diferentes posiciones. Para Heidegger el actuar en un mundo ya constituido es lo prerreflexivo y el fundamento de todo operar humano. Merleau-Ponty ubica este operar prerreflexivo en el cuerpo y Sartre en esa sensación de unidad que siempre nos da la sensación de presencia. Estos debates van a ser pasados por alto en este texto, ya que nos interesa resaltar algunas ideas en común para poder entender de cierta manera la consciencia

4 En la filosofía de Bergson (2006), la vida de consciencia se caracteriza de acuerdo con dos modos en que puede operar: por un lado está el flujo puramente cualitativo de los estados de consciencia; en este plano del flujo temporal los estados se encuentran fusionados los unos con los otros, y no existen en ellos la diferenciación del antes y el después; estas diferenciaciones son las que introduce la segunda modalidad principal de la consciencia, esto es, el modo del pensamiento reflexivo, el cual establece esos aspectos discretos de la experiencia al disecar y espacializar el flujo de consciencia, permitiendo diferenciar un contenido de otro. Las totalidades sensibles a las que se refiere James apuntan a la dimensión puramente cualitativa del fluir temporal, previo a la operación de la actividad diferenciadora de la atención.

5 Se sabe que Köhler y Koffka fueron alumnos de Husserl, y que muchos de sus planteamientos fueron influidos por el padre de la fenomenología. Sin embargo, la influencia fue mutua, pues el mismo Husserl, y algunos continuadores del movimiento fenomenológico (en especial, Merleau-Ponty), incorporaron algunas de las tesis de la escuela de la Gestalt.

6 El tema de las síntesis pasivas de la consciencia concierne, según Husserl, al estudio fenomenológico del 'inconsciente'. Claro está, no se trata del inconsciente freudiano, sino que más bien podría equipararse con lo que Freud denominaba como la esfera de lo pre-consciente, a saber, el dominio de lo mental que prepara el terreno de aquello de lo cual podemos tener una consciencia explícita. Para una clarificación del concepto y la historia de lo inconsciente.

\section{Bibliografía}

BERGSON, Henry

2006 Ensayo sobre los datos inmediatos de la consciencia. Madrid: Sígueme. BROADBENT, Donald

1954 The role of auditory localization in attention and memory span. Journal of Experimental Psychology, 47, 191-196. https://doi.org/10.1037/h0054182

1958 Perception and comunication. London: Pergamon Press.

CARPINTERO, Helio

1996 Historia de las ideas psicológicas. Madrid: Pirámide.

CONWAY, Andrew R.A., COWAN, Nelson \& BUNTING, Michael F.

2001 The cocktail party phenomenon revisited: The importance of working memory capacity. Psychonomic Bulletin \& Review, 8, 331-335. https://doi. org/10.3758/BF03196169

\section{CARRUTHERS, Peter}

2005 Consciousness: essays from a higher-order perspective. New York: Oxford. 
DRIVER, John

2001 A selective review of selective attention research from the past century. British Journal of Psychology, 92, 53-78. Recuperado de: https://bit.ly/3rytAly

JAMES, William

1989 Principios de Psicología. México: Fondo de Cultura Económica.

2000 Pragmatismo: un nuevo nombre para viejas formas de pensar. Madrid: Alianza.

FECHNER, Gustav Theodore

1966 Elements of psychophysics. New York: Rinehart and Winston

GURWITSCH, Aron

1979 El campo de la consciencia: un análisis fenomenológico. Madrid: Alianza

2009 The selected Works of Aron Gurwitsch: Studies in phenomenology and psycho$\log$ (1901-1973). New York: Springer.

HARDY LEAHEY, Tomas

1994 A history of modern psychology. Englewood Cliffs, NJ: Prentice-Hall.

HUSSERL, Edmund

1986 Ideas relativas a una fenomenología pura y una filosofía fenomenológica. México: Fondo de Cultura Económica.

2001 Analyses concerning passive and active synthesis: lectures on transcendental logic. Netherlands: Kluwer Academic Publishers.

KÖHLER, Wolfgang

1929a/1959 Gestalt psychology: an introduction to new concepts in modern psychology. New York: A Mentor Book.

1929b/1967 Psicología de la configuración. Madrid: Morata.

LYCAN, William

1995 Consciousness and experience. Cambridge: MIT Press.

MEINONG, Alexius

1981 Teoría del objeto. México: UNAM Instituto de Investigaciones Filosóficas.

MERLEAU-PONTY, Maurice

1984 Fenomenología de la oercepción. Barcelona: Planeta de Agostini.

O'SHAUGHNESSY, Brian

2002 Consciousness and the World. New York: Oxford University press.

PIAGET, Jean

1976 La toma de conciencia. Madrid: Eds. Morata.

SARTRE, Jean Paul

2006 El ser y la nada. Buenos Aires: Losada.

VYGOTSKY, Lev

2000 El desarrollo de los procesos psicológicos superiores. Barcelona: Crítica.

ZAHAVI, Dan

2005 Subjectivity and selfhood. Cambridge, Mass: MIT Press.

Fecha de recepción de documento: 15 de julio de 2020

Fecha de revisión de documento: 15 de septiembre de 2020

Fecha de aprobación de documento: 15 de noviembre de 2020

Fecha de publicación de documento: 15 de enero de 2021 